\title{
Load spectra and fatigue damage: applications to the automotive industry
}

\author{
Matteo Luca Facchinetti ${ }^{1, *}$ \\ ${ }^{1}$ PSA Groupe, Centre Technique de Belchamp, CC SX.BP.06, 25240 Voujeaucourt, France
}

\begin{abstract}
High cycle fatigue behaviour of materials is historically assessed with constant amplitude and variable amplitude loads, respectively. Thus, a long-lasting debate is extremely active in the academic community, trying to link experimental results coming from these different approaches. Overcoming all this, since the 1970s several industrial fields have been choosing to consider representative customer load spectra (in terms of amplitude, not frequency) as the best way to test both materials and structures. In particular, the automotive industry makes use of specific car loading spectra, regularly fed by the customer knowledge and practised on proving grounds. This paper presents a highlight on such spectra, neglecting any sequence effect of the load time history, thus accepting the Palmgren-Miner's rule as an assumption. Whereas a recent communication on this very topic focused on the basic occurrence spectra, which is absolutely independent from the material properties of the car parts, here we deal with the final damage assessment. Obviously, it is worth knowing which part of the spectrum is mainly responsible for the most relevant fatigue damage.
\end{abstract}

\section{Introduction}

This paper is devoted to the fatigue phenomenon applied to automotive passenger vehicles. More precisely, we focus on the chassis system highlighted in figure 1, which provides basic functions like braking, steering, vertical suspension, vehicle handling ...

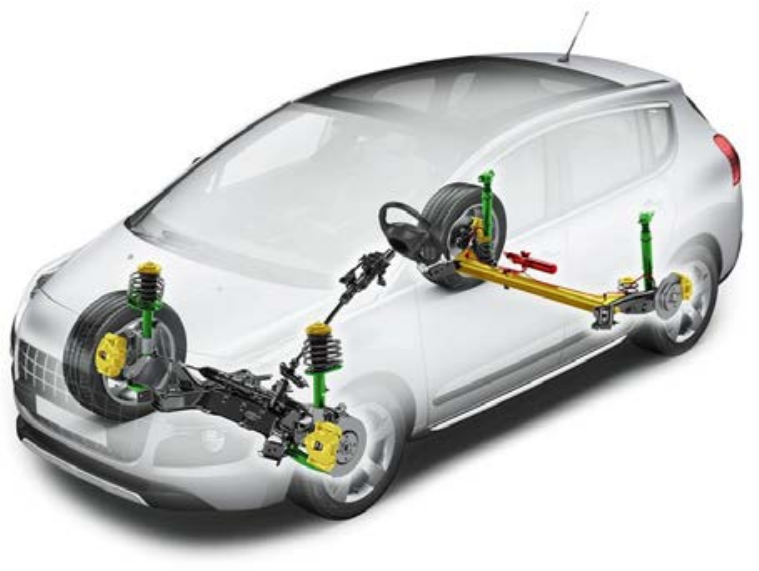

Fig. 1. Passenger car chassis system: front and rear axles.

The chassis system is almost composed of safety parts, whose reliability with respect to fatigue is expected to be proven, by numerical computations and experimental tests, respectively. Fatigue is one of the most relevant design concerns, even if not the only one. In terms of orders of magnitude, considering a life target of about $10^{5} \mathrm{~km}$, the customer usage analysis leads to count up to $10^{6}$ elementary braking and cornering events, i.e. $10^{6}$ cyclic load occurrences, thus calling for high cycle fatigue. For the sake of simplicity, within this framework we pay attention on usual driving situations only, coming from everyday life customer usage, thus neglecting any overload related to special driving conditions or misuse.

Road load time series recorded to feed both numerical computations and experimental tests may be long and complex signals. Whether measured data come from proving grounds or customer usage, engineers are more accustomed to reduce them on more simple signals. This may be achieved thanks to a fatigue damage equivalence process, calling for at least three basic problems:

1. identify load cycles (i.e. sinusoidal patterns of defined mean, amplitude) from a time history

2. take into account for any sequence effect, i.e. the relevance of the cycle load order

3. compute a relevant fatigue damage induced by load cycles of different amplitude (and mean)

For the first step, there is a large consensus since the work of Endo \& al. [1] and the application of the wellknown "rainflow" count method, which has already been published in international standards. We simply accepts this state-of-the-art as an assumption. The second one is still under vigorous discussion: on one hand, there are the advantages and shortcuts of the simplest linear rule proposed by Palmgren and Miner [2,3] nearly 100 years ago; on the other hand, there exists several more recent and complex models for which the material parameters need for complex identifications as well. In this paper,

Corresponding author: matteoluca.facchinetti@mpsa.com 
we just assume and apply the simplest model: please remember that the in-service load history of a passenger car is very long (the order of magnitude is of about 10 years) and somewhat random, from customer to customer and with respect to any possible normal usage operating conditions. At last, the fatigue response may be sensible to the load time history, the gap between variable and constant amplitude points of view resulting in a longlasting debate among the fatigue community. This paper humbly presents some insights between two frameworks applied by the automotive industry in France and Germany, respectively.

On one hand, the pioneering Wöhler model [4] is the natural reference when dealing with constant amplitude loading and represents the historical point of view of French engineers. The relevant fatigue damage equivalent signal corresponds to a single sinusoidal wave of given amplitude (and frequency), the simplest one to be managed by a testing rig or a FE solver.

On the other hand, standard load spectra are widely applied since the seminal work of Gassner [5] and applied by German engineers since at least 50 years. Here the relevant fatigue damage equivalent signal is composed by blocks of sinusoidal waves of different amplitudes and occurrences, see figure 3 .

The key features beyond this apparently nothing but theoretical discussion are practical indeed: it is matter of effective fatigue assessment of chassis parts through numerical computations and experimental tests, quotation of the relevance of a proving ground, acceleration of testing procedures ...

In the sequel of a previous recent communication on this very topic [6], this paper is organized as follows. At first, we announce all the assumptions and describe the basic models used $(\$ 2)$. Then, a historical overview on standard load spectra is recalled from the literature, with particular interest on a particular load spectrum family (\$3). Thus, special attention is devoted to the fatigue damage assessment $(\$ 4)$. At last, discussions and conclusions end the paper $(\S 5)$.

\section{Assumptions and models}

We recall here the main assumptions already presented in [6]. The framework is that of high cycle fatigue phenomenon [7,8], which applies cyclic loads under the yield threshold of the materials used for chassis system parts. Nowadays it is of common practice to represent the load cycle amplitude, $S$ with respect to the life cycle number, $N$, see figure 2 . Following Wöhler [4], constant amplitude fatigue tests provided up to final failure (or any other relevant stop criterion) lead to the so-called Wöhler curve, see figure 2. One of the basic models used to express a relationship between the load cycle amplitude, $\mathrm{S}$ with respect to the life cycle number, $N$ is that of Basquin [9], as expressed by the following equation:

$$
N S^{b}=B
$$

where the Basquin slope, $b(b>1)$ and the Basquin constant, $B(B>>1)$ are material parameters to be identified. At least for metallic materials, this model is supposed to hold from $10^{4}$ up to $10^{6}$ cycles and leads to a linear relation in the $\log S-\log N$ plane, see figure 2. For some reference values of the Basquin slope and a more comprehensive statistical approach, please refer to [10].

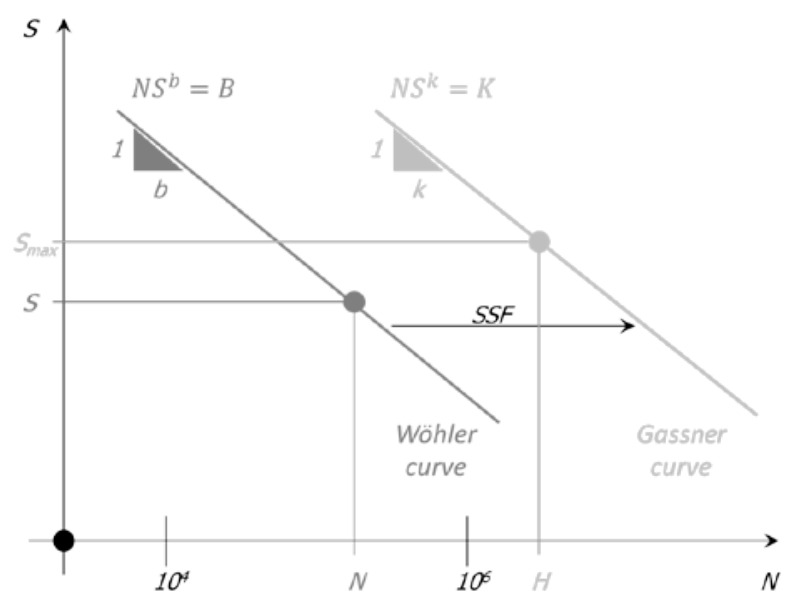

Fig. 2. Wöhler curve (left, dark grey) \& Gassner curve (right, light grey).

Moreover, Ernst Gassner seminal work [5] introduces the concept of load spectrum in order to define a variable amplitude lifetime. Please note that the term "spectrum" does not rely here to any frequency analysis, but only deals with the occurrence of load cycles with different amplitudes within the same load sequence. Actually, the load frequency is not expected to have any effect in the range of the load time histories of interest. The original Gassner block schedule, showed in figure 4 , is composed of eight load amplitude levels, $S_{i}$ linearly distributed from the lower, $S_{\min }$ up to the maximum, $S_{\max }$ and associated to cycle numbers $N_{i}$ resulting from a Gaussian-like distribution. A cumulative occurrence, $H$ may be introduced by the following equation:

$$
H_{i}=\sum_{j=1}^{i} N_{j}
$$

where the summation is usually started from the maximum amplitude load level (i.e. $S_{l}=S_{\max }$ ), which is expected to be associated to the smallest occurrence (i.e. $N_{1}=N_{\min }$ usually chosen to be equal to 1). Thus, applying the summation over the whole load sequence leads to the spectrum length, $H$ which actually counts the overall cycle number of the spectrum, no matter of the load amplitudes. From the experimental point of view, once the load spectrum pattern is established by the choice of $S_{1}=S_{\max }$, the load sequence is applied again and again until the specimen failure (or any other 
relevant stop criterion), leading to the overall lifetime $H$. Within the previous $S-N$ plane (actually $\log S-\log N$ ), Gassner reports the experimentally achieved overall lifetime, $H$ at the maximum load amplitude, $S_{\max }$ of the load spectrum considered, leading to the so-called Gassner curve, see figure 2. Another Basquin-like relation may be used to model Gassner results:

$$
H S_{\max }^{k}=K
$$

where the slope, $k$ and the constant, $K$ are material parameters to be identified.

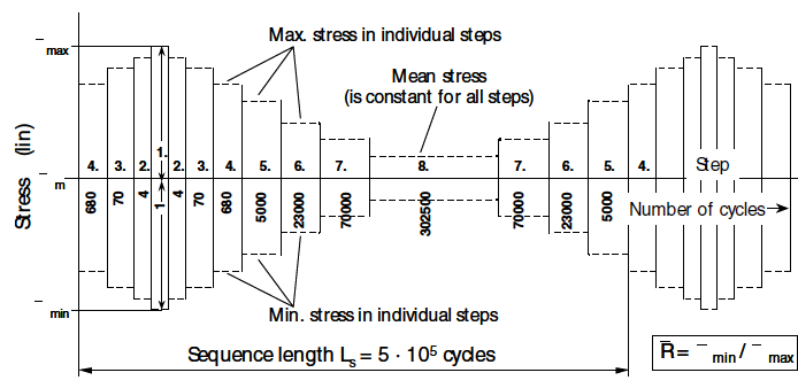

Fig. 3. Gassner load sequence - courtesy from [13].

Several assumptions apply within the Gassner framework. At first, let us recall that the whole spectrum is supposed under the yield threshold of the materials used. Secondly, the load sequence, i.e. the way all the load cycles of different amplitudes are mixed before to be applied to the specimen, is expected to minimize any history effect: please note that since the Gassner original block sequence, see figure 4 , both increasing and decreasing load amplitudes are considered within the basic load schedule. Moreover, the sequence length, $H$ should be at least one order of magnitude smaller than the expected lifetime, in order to accept the concept of an elementary spectrum as a relevant metrics. If it were not the case, this would imply that rare maximum load levels are not guaranteed to be applied with the same statistical representation than any other lower load levels, so that fatigue damage would be too sensible to them.

Since the linear Palmgren-Miner's damage rule $[2,3]$ is assumed, it may be proven [11] that the exponents of the Basquin and Gassner laws are expected to be exactly the same:

$$
b=k
$$

Actually, if no history effects are considered, the only material parameters in use are the pairs $(B, b)$ and $(K, k)$. Whereas the intercepts $B$ and $K$ are associated to the choice of plotting options ( $S$ or $S_{\max }$ and $N$ or $H$, respectively), the slopes $b$ and $k$ are expected to describe the same physical behavior: thus they must have the same value.

At last, please note that plotting Gassner lines $\left(S_{\max }\right.$; $H$ ) in the Wöhler plane $(S ; N)$, see again figure 1 , gives the graphical illusion that the Gassner framework offers longer lifetime than Wöhler does. This is nothing but a graphical mirage, due to the choice of $S_{\max }$ as the relevant load amplitude for the Gassner plot. According to [12], it may be proven [11] that the horizontal shift of the Gassner line with respect to the Wöhler one is provided by the following formula:

$$
S S F=\log \left[\frac{\sum N_{i}}{\sum N_{i}\left(\frac{S_{i}}{S_{\max }}\right)^{1 / b}}\right]
$$

where SSF stands for spectrum shape factor, which solely depends on the shape of the spectrum and the Basquin slope. Please note that if the spectrum collapses to a constant amplitude load (i.e. $S_{i}=S_{\max }$ ), the Gassner framework reduces to the original Wöhler one: $S S F=0$. For any consistent variable amplitude spectrum, $S S F$ is definite positive, because of $b>1$ and $S_{i} / S_{\max }<1$. It may be shown that $S S F<1$ for spectra with many large and few small cycles, whereas SSF $>1$ for spectra with larger occurrences for small amplitude cycles. For example, for $b=5$ and a Gaussian-like load spectrum as the original Gassner one, one has SSF $\sim 2.5$, which means an horizontal shift of more than 2 decades on the right with respect to the Wöhler curve.

\section{Standard load spectra}

Both European and US working groups have been providing reference design load spectra or standard load time-histories since the 1970s, respectively for the aeronautics, the energy industry, the automotive ... along with experimental test results and fatigue criteria. Heuler $\&$ al. $[12,13,14,15]$ published a comprehensive review where each cited example is collected with its own purpose, the intended structural application, a brief qualification, and some more technical details like the block size.

In order to go further with the comparison and analysis of load spectra, it may be useful to provide a general mathematical formulation. According to $[13,14]$, we make use of the following equation:

$$
\frac{\log H_{i}}{\log H_{0}}=1-\left(\frac{S_{i}}{S_{\text {max }}}\right)^{v}
$$

where the pattern of the spectrum is based on an exponential law and $v$ is the sole spectrum parameter, called the shape exponent. This equation may be plotted in a dimensionless field, see figure 4, considering the amplitude load ratio $r_{i}=S_{i} / S_{\max }$ on the Y-axis and the occurrences ratio $\log H_{i} / \log H_{0}$ on the X-axis. 


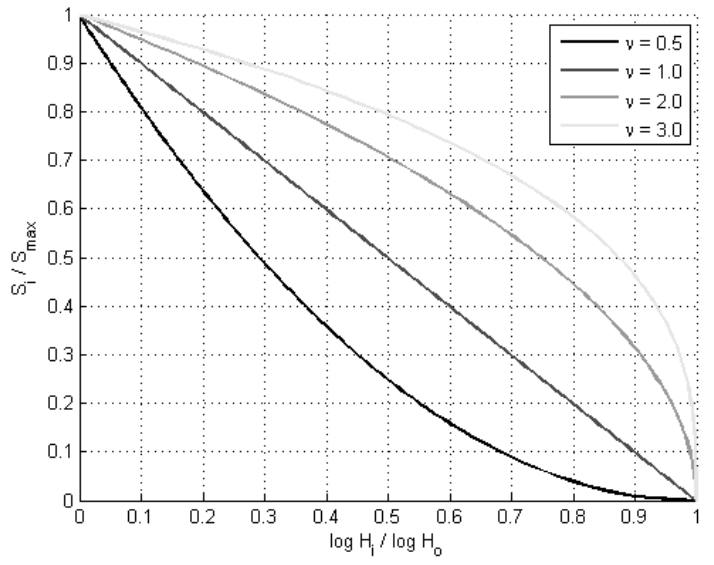

Fig. 4. Standard load spectra according to $[13,14]$ - cumulative occurrences, $H$.

Switching from cumulative occurrences, $H_{i}$ to usual cycle number, $N_{i}$, thus making use of equation (2) on the other way round, leads to the more explicit figure 5. For the sake of precision, whereas figure 4 is full dimensionless, figure 5 needs to define the overall spectrum length, $H_{o}$ here assumed equal to $10^{6}$.

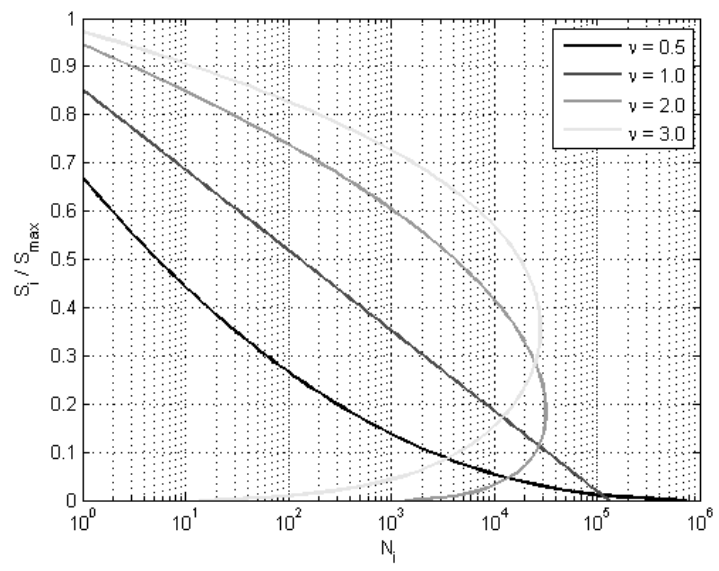

Fig. 5. Standard load spectra according to $[13,14]$ - effective occurrences, $N$.

Figure 5 allows us to give a physical meaning at the shape parameter, $v$. A unit value is associated to a linear pattern (please note that abscissa is in log scale), so that $v$ $<1$ corresponds to $\partial^{2} N / \partial S^{2}<0$ and a concave spectrum, for which the lower the load, the greater is the occurrence, whereas $v>1$ corresponds to $\partial^{2} N / \partial S^{2}>0$ and a convex spectrum, for which the larger occurrence is not associated to the weakest load anymore. It should be noted that figure 5 shows an apparently odd behavior at the upper left corner: the expected maximum load seems to be associated to less than one cycle number, as the curves vanish on the left before attending the maximum load level. Actually, the proposed spectrum model states that the maximum amplitude $S_{I}=S_{\max }$ always corresponds to a unit cycle indeed $N_{\min }=1$. Then, according to the shape imposed by $v$, the following load level expected to have more than 1 cycle is at the intercept between the spectrum curve with the vertical axis at unit abscissa.

Please note that the shape exponent does not depend on the material fatigue parameters like the Basquin slope, because of it results from the cycle count operation prior to any damage ponderation. Thus, since the formulation (6) is accepted, the identification of the shape exponent, $v$ may be a key to compare load spectra coming from independent sources, histories and practices. According to $[13,14]$, typical values of the shape exponent may be identified and linked to the SSF, equation (5) for a standard Basquin parameter value, $\mathrm{b}=$ 5 , as reported in table 1.

Table 1. Typical load spectra, according to $[13,14]$.

\begin{tabular}{|c|c|c|}
\hline$v$ & $\begin{array}{c}\text { SSF } \\
(\mathbf{b}=\mathbf{5})\end{array}$ & description \\
\hline$\infty$ & 0 & $\begin{array}{c}\text { constant } \\
\text { amplitude }\end{array}$ \\
\hline 4 & 1.5 & $\begin{array}{c}\text { convex } \\
\text { spectrum }\end{array}$ \\
\hline 2 & 2.5 & $\begin{array}{c}\text { Gaussian } \\
\text { spectrum }\end{array}$ \\
\hline 1 & 3.8 & $\begin{array}{c}\text { linear } \\
\text { spectrum }\end{array}$ \\
\hline$<1$ & 4.2 & $\begin{array}{c}\text { concave } \\
\text { spectrum }\end{array}$ \\
\hline
\end{tabular}

In a recent paper [6], a comparison between load spectra applied for the automotive has been proposed, based on the identification of the shape exponent, $v$ and thus limited to the basic occurrence spectra, which is absolutely independent from the material properties. At present, in the following section we focus on the fatigue damage assessment, so that the Basquin slope, $b$ is the second relevant parameter to be taken into account.

\section{Damage assessment}

In the previous section we described a load spectra family already proposed in the literature [13,14], equation (6), and recalled its main characteristics. We now introduce a damage norm, according to the Basquin model, equation (1):

$$
D=N S^{b}=D(v, b)
$$

and wish to highlight how the fatigue damage is partitioned through the load spectrum, as a function of both the spectrum shape, $v$ and the Basquin slope, $b$. As already commented in [16], figure 6 shows the dimensionless damage distribution of the considered spectra family for an usual value of the Basquin slope, $b$ $=5$. 


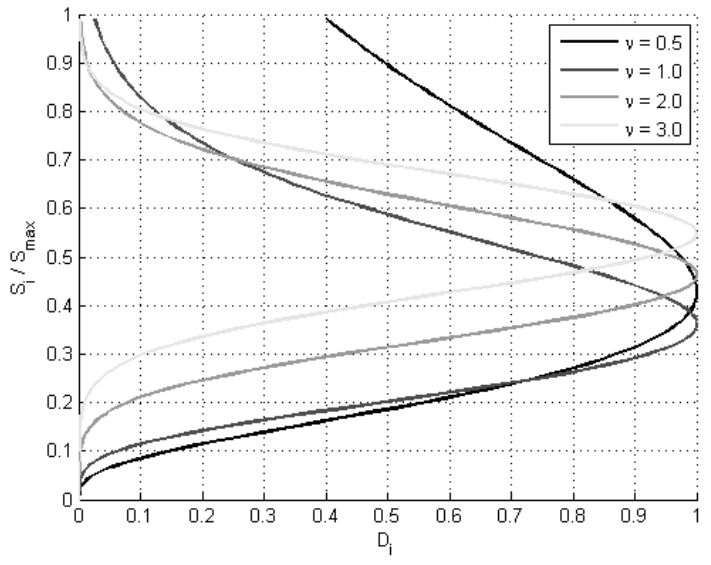

Fig. 6. Standard load spectra according to $[13,14]$ - damage, $D$.

The maximum relative damage is located neither at the maximum load level, nor the minimum one, but somewhere in the middle, not so far from the central kernel of the spectra. On one hand, lower loads than the maximum damage one are candidates for omission, as they may not provide significant damage and are associated to the higher occurrences. On the other hand, higher loads than the maximum damage one may be responsible for lower damage, but are associated to the lower occurrences indeed, so that any omission is more sensible. Depending on the shape exponent, the load level of maximum damage is shown to move upwards as the shape exponent increases. In a more comprehensive view, according to figure 6 , the value of the dimensionless load amplitude generating the maximum damage may be defined by:

$$
S_{\max D}=\frac{S_{i}}{S_{\max }}\left(\frac{\partial D}{\partial S}=0\right)
$$

which is a function of the Basquin slope, $b$ and the shape exponent, $v$. Figure 7 presents a contour plot of $S_{\max D}$, where $b$ spans from 3 to 13 and $v$ from 0.5 to 3.5 .

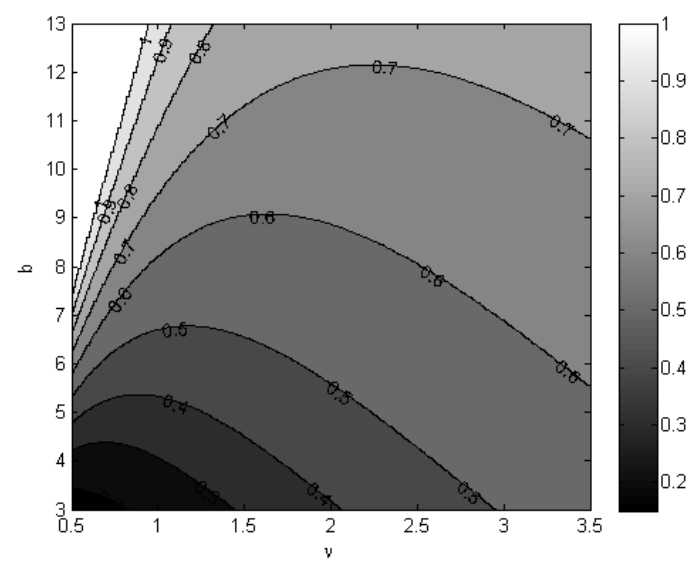

Fig. 7. Load amplitude, $S_{\max D}$ generating the maximum damage as a function of the Basquin slope, $b$ and the shape exponent, $v$.
Most of the contour plot is bounded within 0.3 and 0.7 , i.e. the load amplitude associated to the maximum damage lies in the $0.5 \pm 0.2$ range. Significant deviations appear only for Basquin slopes far from the median value, $b=8$ or low shape exponent, $v<1$. Please note that the upper left corner may be a critical one indeed, because of the relevant damage of the overall spectrum is provided by its few higher amplitude load cycles only. For a given spectrum shape, i.e. a fixed value of $v$, an increasing value of the Basquin slope leads to a higher relevant load amplitude. Whereas for a given Basquin slope, $b$ an increasing value of the shape exponent, $v$ leads to a monotonic decrease of the relevant load amplitude only for lower Basquin slopes.

$S_{\max D}$ provides useful information on the damage content of a spectrum, but does not takes into account what happens just around it. This gap may be closed by introducing the concept of "damage barycentre", i.e. a weighted load with respect to damage [17], defined by:

$$
S_{\text {aveD }}=\frac{\sum_{i} \frac{S_{i}}{S_{\max }} D_{i}}{\sum_{i} D_{i}}
$$

From a purely statistical point of view, $S_{\text {aveD }}$ represents an overall average, whereas $S_{\max D}$ is a mode of the damage distribution. $S_{\text {ave }}$ is again a function of the Basquin slope, $b$ and the shape exponent, $v$. Figure 8 presents a contour plot of $S_{\text {ave }}$, where $b$ spans from 3 to 13 and $v$ from 0.5 to 3.5 .

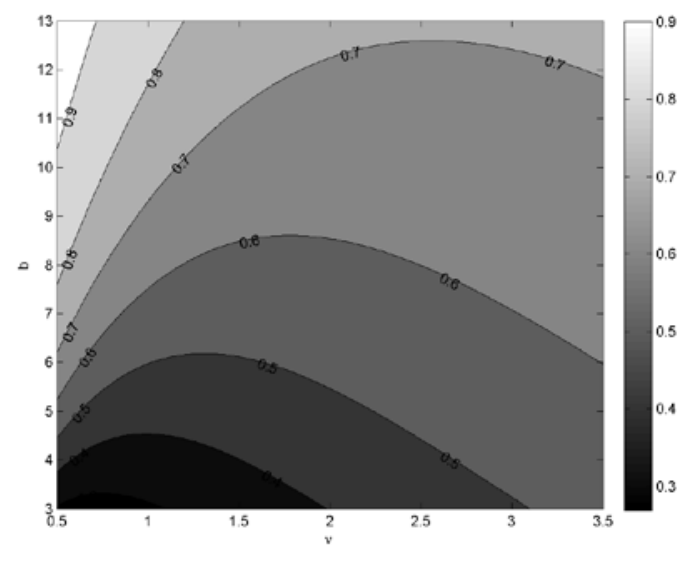

Fig. 8. Load amplitude, $S_{\text {ave } D}$ generating the maximum damage as a function of the Basquin slope, $b$ and the shape exponent, $v$.

The comparison of figures 7 and 8 shows that the damage barycentre, $S_{\text {ave } D}$ and load generating the maximum damage, $S_{\max D}$ are almost the same. If $v$ is larger than the unit value, i.e. for convex spectra, the scatter between $S_{\max D}$ and $S_{\text {aveD }}$ is lower than 5\%. Only for concave spectra the scatter may rise up to $10 \%$ or more, especially for higher values of the Basquin slope. Roughly speaking, as far as the damage distribution is bell-like, no significant difference is expected between the average and the mode. 


\section{Conclusions and outlooks}

This paper is devoted to the fatigue phenomenon applied to automotive passenger vehicles. At first (\$2), we announced all the assumptions and described the basic models applied in the high cycle fatigue framework. Then ( $\S 3)$, a historical overview on standard load spectra has been recalled from the literature, with particular interest on a particular load spectrum family, equation (6). Thus (§4), a special focus has been provided to the fatigue damage assessment, going further than a previous paper [6].

Let us recall here the main hypothesis of this work.

1. The fatigue damage is based on the Basquin model, applying a unique slope $b$ on the overall load domain. This provides the first relevant parameter, whose significant values may be found in the literature, e.g. in [10]. For fatigue design of chassis parts, a particular interest is usually devoted to lower values, i.e. from 3 to 5 , when dealing with welded assemblies and notched parts.

2. A spectrum family is chosen according to $[13,14]$. This provides the second relevant parameter, the shape exponent, $v$ for which standard values are already available for several industrial applications $[12,13,14]$. For fatigue design of chassis parts, some values of interest are reported in [6].

Within this framework, the damage assessment may be highlighted by the analysis herein developed, figures 5 to 8 , and the use of the relevant dimensionless loads $S_{\max D}$ and $S_{a v e D}$, equations (8) and (9), respectively.

The present analysis may be easily extended to any other spectra family than (6). Actually, the choice of the underlying mathematical model and its physical meaning should deserve more discussion. Moreover, a full mathematical approach may also be considered. Finding extreme values and locations of continuous functions should help to get more general properties.

This work began from fruitful discussions between German \& French automotive researchers within the framework of the DVM - Deutscher Verband für Materialforschung und prüfung \& SF2M - Société Française de Métallurgie et des Matériaux. Moreover, the author wishes to acknowledge the IRDL laboratory at ENSTA Bretagne (Brest), whose researchers and students contributed to this work during several internships.

\section{References}

1. M. Matsuiski, T. Endo, Fatigue of metals subjected to varying stress, Japan Society of Mechanical Engineering (1969)

2. A. Palmgren, Die Lebensdauer von Kugellagern The Fatigue Life of Ball-Bearings, Zeitschrift des Vereins Deutscher Ingenieure (1924), 68, 339-341 (in German)

3. M.A. Miner, Cumulative damage in fatigue, Journal of Applied Mechanics (1945), 12, A159A164

4. A. Wöhler, Versuche zur Ermittlung der auf die Eisenbahnwagenachsen einwirkenden Kräfte und die Widerstandsfähigkeit des Wagen-Achsen, (1860), Zeitschrift für Bauwesen, X, 583-616 (in German)

5. E. Gassner, Festigkeitsversuche mit wiederholter Beanspruchung im Flugzeugbau - Strength tests under repeated loading for aeronautical engineering Luftwissen (1939). 1939;6:61-4 (in German)

6. M. Facchinetti, Fatigue damage of materials and structures assessed by Wöhler and Gassner frameworks: recent insights about load spectra for the automotive, Procedia Engineering (2017) 213, 217-225

7. C. Bathias, J.P. Baïlon, La Fatigue des matériaux et des structures - Fatigue of materials and structures, Hermes Paris (1997) (in French)

8. S. Suresh, Fatigue of Materials, Cambridge Solid State Science Series (1998)

9. O.H. Basquin, The exponential law of endurance tests, proceedings of the ASTM (1919), 30, 625

10. S. Bergamo, P. Schimmerling, F. Triboulet, P. Wilson, M.L. Facchinetti, M. Monin, F. Lefebvre, B. Weber, Préconisations pour les caractéristiques statistiques de résistance en fatigue - Applications aux aciers et autres matériaux utilisés dans la construction automobile - SIA (2017) (in French) http://www.sia.fr/publications/494-preconisationspour-caracteristiques-statistiques-resistancefatigue? lng=fr

11. L. Navrátil, Fatigue under variable amplitude load, internship report, ENSTA Bretagne, Brest (2016)

12. C. Berger et al., Betriebsfestigkeit in Germany - an overview, International Journal of Fatigue (2002) $24,603-625$

13. P. Heuler, T. Bruder, H. Klätschke, Standardised load-time histories - A contribution to durability issues under spectrum loading, Materialwissenschaft und Werkstofftechnik. (2005), 36, $669-677$

14. P. Heuler, H. Klätschke, Generation and use of standardised load spectra and load-time histories, International Journal of Fatigue (2005) 27, 974-990

15. C.M. Sonsino, Fatigue testing under variable amplitude loading, International Journal of Fatigue (2007), 29, 1080-1089

16. M. Martins de Oliveira, Dimensionnement à la fatigue des éléments d'une Liaison $\mathrm{Au}$ Sol, internship report, ENSTA Bretagne, Brest (2018)

17. I. Raoult, B. Delattre, private communication 